\title{
Neural Network Based Active Disturbance Rejection Control of a Novel Electrohydraulic Servo System for Simultaneously Balancing and Positioning by Isoactuation Configuration
}

\author{
Qiang Gao, ${ }^{1}$ Yuanlong Hou, ${ }^{1}$ Kang Li, ${ }^{1}$ Zhan Sun, ${ }^{2}$ Chao Wang, ${ }^{1}$ and Runmin Hou ${ }^{1}$ \\ ${ }^{1}$ School of Mechanical Engineering, Nanjing University of Science and Technology, Nanjing 210014, China \\ ${ }^{2}$ Institute of North Automatic Control Technology, Taiyuan 030006, China \\ Correspondence should be addressed to Qiang Gao; gaoq0916@sina.com
}

Received 12 June 2015; Revised 1 September 2015; Accepted 2 September 2015

Academic Editor: Mario Terzo

Copyright (C) 2016 Qiang Gao et al. This is an open access article distributed under the Creative Commons Attribution License, which permits unrestricted use, distribution, and reproduction in any medium, provided the original work is properly cited.

To satisfy the lightweight requirements of large pipe weapons, a novel electrohydraulic servo (EHS) system where the hydraulic cylinder possesses three cavities is developed and investigated in the present study. In the EHS system, the balancing cavity of the EHS is especially designed for active compensation for the unbalancing force of the system, whereas the two driving cavities are employed for positioning and disturbance rejection of the large pipe. Aiming at simultaneously balancing and positioning of the EHS system, a novel neural network based active disturbance rejection control (NNADRC) strategy is developed. In the NNADRC, the radial basis function (RBF) neural network is employed for online updating of parameters of the extended state observer (ESO). Thereby, the nonlinear behavior and external disturbance of the system can be accurately estimated and compensated in real time. The efficiency and superiority of the system are critically investigated by conducting numerical simulations, showing that much higher steady accuracy as well as system robustness is achieved when comparing with conventional ADRC control system. It indicates that the NNADRC is a very promising technique for achieving fast, stable, smooth, and accurate control of the novel EHS system.

\section{Introduction}

Large pipe weapons, arms of heavy machineries, industrial robotics, and space-borne manipulators possess a sort of long and heavy linkages which have high ratio of length to diameter and time-varying unbalancing torque induced by the misalignment between its gravity center and the corresponding trunnion. The unbalancing torque serving as a sort of strong disturbance highly deteriorates control performances of the system $[1,2]$.

Currently, there are two kinds of system balancing strategies, namely, the external force based system balancing and the internal driving based system balancing. The external methods mainly depended on adopting proper balance weights or adopting balance machinery, and the static unbalancing components can be well compensated. Practically, the unbalancing part including partial static and dynamic unbalancing components was treated as external disturbance and further compensated during active control of the system. Regarding the large diameter of the pipe, the unbalancing components were heavy, highly challenging the control system for positioning [3, 4]. Moreover, the extra machinery for balancing strongly increased weights and costs of mechanical systems, being difficult to meet the lightweight requirements. With the internal driving based system balancing methods, the most directly way was to use the driving forces for system balancing $[5,6]$. Generally, the currently adopted method based on direct driving was essentially a feedforward inverse compensation strategy, and it cannot achieve real-time balancing. It was obvious that not 
enough driving forces can be provided by the motor servo system in terms of the heavy pipes. Another kind of adaptive balancing method combining the hydraulic accumulator and balancing machinery was recently developed in [7]. However, only partial unbalancing forces can be compensated during the process. Also, this method cannot be applied for active rejection of the unbalancing forces during the positioning.

As discussed above, active compensation for the unbalancing components in pipe positioning is still an outstanding issue. In the present study, a novel electrohydraulic servo (EHS) system where the hydraulic cylinder possesses three cavities is developed. In the EHS system, the balancing cavity of the EHS is especially designed for active compensation for the unbalancing force of the system, whereas the driving cavity is employed for real-time positioning and disturbance rejection of the large pipe. By means of the hydraulic cylinder with three cavities, simultaneous control and disturbance rejection can be achieved by using only one driving source, namely, isoactuation, greatly reducing weights of weapons with heavy pipes. However, there are certain extremely complicated segments with strong nonlinearities in gun control systems, including time-varying parameters induced by varying working conditions, random external applied loads, and complex friction forces between the cannon and trunnion. All these nonlinear behaviors add difficulties in achieving high control performances (both static and dynamic), blocking improvements of working performances of the gun control system. In terms of nonlinear control, considerable work has been done based on certain types of adaptive control strategies, such as fuzzy control, adaptive sliding mode robust control, and adaptive equivalent disturbance compensation control [4-7]. All these nonlinear control methods can significantly improve the uncertainties of the control system in terms of its tolerance and robustness, but only at the expense of the positioning accuracy and response speed.

The recently developed active disturbance rejection control (ADRC) is an efficient nonlinear digital control strategy which regards the unmodeled part and external disturbance as overall disturbance of the controlled system [8-10]. The ADRC mainly consists of a tracking differentiator (TD), an extended state observer (ESO), and a nonlinear state error feedback (NLSEF) control law. In this ADRC approach, the processes with higher orders, uncertainties, and unmodeled dynamics are viewed as lower-ordered systems with general disturbances; meanwhile, the general disturbances are estimated by ESO and are actively compensated [11-20]. There are a large number of adjustable parameters in the ADRC, and choosing proper parameters can contribute to excellent performances of the control system. To optimally get the system parameters, the global optimization strategy based offline tune methods $[11,12]$ and the artificial intelligent based online tune methods [13-16] were developed. Generally, the off-line tune methods are highly dependent on identified physical model of the controlled system, and the obtained parameters cannot adapt to variable working conditions. The online updating method can adjust control parameters to get optimal control performance in real time. In [13], the fuzzy control scheme was introduced in the ADRC to estimate the states and accordingly update the compensation factors of the ESO. In [14], the diagonal recurrent neural network was introduced to realize online tuning of the NLSEF. In terms of parameters of the NLSEF, the compensation gains in ESO highly affect estimation performance of the control system, especially the estimation accuracy of overall disturbances. Thereby, the backpropagation neural network (BPNN) was adopted in [15] to tune the gains in ESO. However, the BPNN suffers easily trapping in local minimum and slow convergent speed.

Motivated by this, the radial biases function (RBF) neural network is introduced in an improved ADRC to solve the practical issue in control of the newly developed EHS system with three hydraulic cavities for simultaneous balancing and positioning, constructing the novel neural network based active disturbance rejection control (NNADRC) strategy. Four adjustable compensation gains in the ESO are online updated by adopting the RBF neural network.

\section{The Isoactuation Electrohydraulic Servo System}

2.1. System Configuration and Working Principle. Figure 1 illustrates the schematic of the isoactuation EHS system for simultaneous balancing and positioning. As shown in Figure 1, the EHS system mainly consists of a positioning controller, a variable capacity pump, a proportional servo valve, a rotary transformer, a RDC modular, a balancing controller, a constant displacement pump, a proportional pressure-reducing valve, a pressure sensor, and an actuation hydraulic cylinder. The actuation hydraulic cylinder has three cavities, namely, the upper, lower, and balancing cavity. A combination of the upper and lower cavities is adopted for system actuation.

In practice, the balancing controller compares and calculates the deviation between the measured and desired pressure in the balancing cavity. The control signal for the proportional pressure-reducing valve is obtained based on the deviation to accurately control the pressure in the balancing cavity, accordingly cancelling the load and weight torque on the system. As for the positioning, the deviation between the measured and desired positions of the pipe is similarly calculated, and accordingly a control signal is generated for the proportional servo valve to control the flow rate and direction of the hydraulic fluid for the upper and lower cavities. Thereby, position of the pipe can be accurately controlled by adopting the feedback control strategy.

2.2. Modelling the EHS System. Assume the state variables are $x=\left[\begin{array}{lll}x_{1} & x_{2} & x_{3}\end{array}\right]^{T}$ with $x_{1}=\theta, x_{2}=\dot{\theta}$, and $x_{3}=\ddot{\theta}$; the state space equation for the isoactuation EHS system can be determined by

$$
\begin{aligned}
& \dot{x}_{1}=x_{2}, \\
& \dot{x}_{2}=x_{3}, \\
& \dot{x}_{3}=f(x)+g u_{1}+d(t),
\end{aligned}
$$




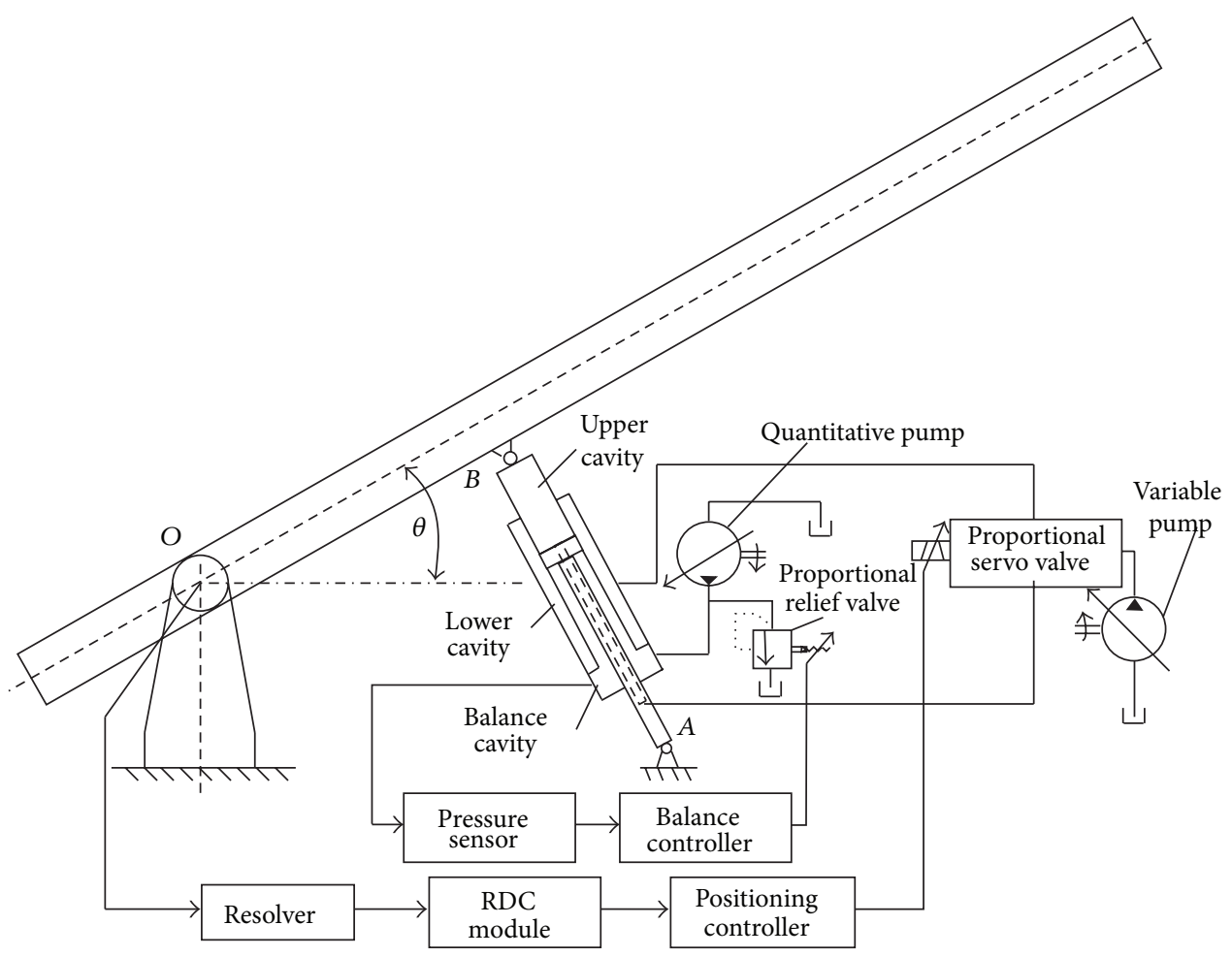

FIGURE 1: Schematic of the isoactuation EHS system.

where

$$
\begin{gathered}
f(x)=-\frac{\beta_{e} C_{t} G}{J V_{0}} x_{1}-\frac{\beta_{e}}{J V_{0}}\left(D_{e}^{2}+C_{t} B_{m}+\frac{V_{0}}{\beta_{e}} G\right) x_{2}, \\
-\frac{\beta_{e}}{J V_{0}}\left(J C_{t}+\frac{V_{0}}{\beta_{e}} B_{m}\right) x_{3}, \\
g=\frac{\beta_{e}}{J V_{0}} D_{e} K_{a} K_{1}, \\
d(t)=\frac{\beta_{e} C_{t}}{J V_{0}} T_{L}+\frac{1}{J} \dot{T}_{L}, \\
|d(t)| \leq \text { Const, }
\end{gathered}
$$

where $\theta$ denotes the actual position of the pipe, $u_{1}$ denotes the output signal of the positioning controller, $\beta_{e}$ is the effective bulk modulus of elasticity, $C_{t}$ is the overall leakage coefficient, $G$ represents the load elastic stiffness, $J$ is the rotational inertia, $V_{0}$ is the volume of a cavity, $D_{e}$ is the equivalent surface total displacement, $B_{m}$ is the viscoelastic damping coefficient, $K_{1}$ represents the current-flow rate amplification ratio, $K_{a}$ is the gain of the servo amplifier, and $T_{L}$ represents the external loads. Practically, the overall leakage coefficient, the viscoelastic damping coefficient, the equivalent surface total displacement, and the external loads vary with respect to working conditions, showing strong nonlinear behavior of the working system.

\subsection{Controlling Principle of the EHS System}

2.3.1. Principle of Balancing Control. In (1), the external load $T_{L}$ denotes a combination of unbalancing torque, external disturbance torque, and launch impact torque. To compensate for these external disturbances, a novel active actuation configuration based on a hydraulic cylinder with three cavities is proposed for simultaneous positioning and balancing. The basic principle can be summarized as follows: the weight torque varies with respect to the rotation position $\theta$, which can be expressed by $T_{G}(\theta)$. With a specified arm of force $l(\theta)$ and the acting area $A$, the required pressure $P_{G}(s)$ provided by the balancing cavity can be accordingly determined. To actively balance the weight torque, the pressure in the balancing cavity is controlled by means of the proportional pressure-reducing valve. Thereby, the required torque for balancing the weight torque can be achieved.

Since the working frequency of the proportional pressure-reducing valve is much higher than the natural frequency of its actuation, it can be simply regarded as a proportional element. Thus, the relationship between the control signal $U_{2}(s)$ and output flow rate $Q_{1}(s)$ of the valve can be expressed by

$$
Q_{1}(s)=K_{2} K_{q 1} U_{2}(s),
$$

where $K_{2}$ is the voltage-displacement coefficient and $K_{q 1}$ is the flow rate gain for the valve. 
The theoretical pressure in the balancing cavity can be determined by

$$
\begin{aligned}
& P_{p}(s)=\frac{1}{\left(V_{1} / \beta_{e}\right) s+C_{L 1}}\left[Q_{s 1}(s)-Q_{L 1}(s)-Q_{1}(s)\right] \\
& =\frac{1}{\left(V_{1} / \beta_{e}\right) s+C_{L 1}}\left[Q_{s 1}(s)-\frac{1}{A l(\theta)} s \theta(s)\right. \\
& \left.\quad-K_{2} K_{q 1} U_{2}(s)\right]
\end{aligned}
$$

where $Q_{s 1}(s)$ and $Q_{L 1}(s)$ denote the flow rate of the constant displacement pump and the flow rate into the balancing cavity, $V_{1}$ is the volume between the output end of the pump, the input end of the valve, and the balancing cavity, and $C_{L 1}$ is the leakage coefficient of the balancing cavity.

2.3.2. Principle of Positioning Control. The feedback control scheme is employed for the positioning. Since there are certain nonlinear components and unbalanced torque in the system, the ESO is further employed for the estimation of these unbalanced components; the control signal is then obtained by following the nonlinear state error feedback control law with consideration of the disturbance compensation.

\section{Design of the Controllers}

3.1. Balancing Controller. The typical PID controller is adopted for the balancing of the gun control system. Assume that the desired and practical pressures in the balancing cavity are $P_{G}(t)$ and $P_{p c}(t)$, respectively; the command signal for the balancing system can be determined by

$$
\begin{aligned}
& u_{2}(t)=U_{m}-\left[k_{p} e_{p}(t)+k_{i} \int_{0}^{t} e_{p}(t) d t+k_{d} \frac{d e_{p}(t)}{d t}\right], \\
& e_{p}(t)=P_{G}(t)-P_{p c}(t),
\end{aligned}
$$

where $U_{m}$ denotes the maximum output voltage for the control and $k_{p}, k_{i}$, and $k_{d}$ are proportional, integral, and differential coefficients of the PID controller.

3.2. The Improved ADRC Controller. To better track the trajectory of the control system, an improved ADRC control strategy is developed. To suppress the inherent chatter phenomenon, a novel nonlinear function $\mathrm{nfal}(\cdot)$ featuring smooth switching behavior is employed to replace the conventionally adopted nonlinear function fal $(\cdot)$ which is a key component of the ADRC controller. Besides, the radial biases function $(\mathrm{RBF})$ neural network is adopted to online update the compensation gains of the ESO in the ADRC.

3.2.1. Configuration of the Control System. Schematic of the ADRC is illustrated in Figure 2; it mainly consists of a TD, an ESO, and a NLSEF control law. In this ADRC approach, the processes with higher orders, uncertainties, and unmodeled dynamics are viewed as lower-ordered systems with general disturbances which are further estimated by ESO and are actively compensated.
With the TD process, it is employed for transient process and command signal generation. Fast tracking without overshoots as well as suspension of rapid fluctuations of the control signal when the presetting parameters are suddenly changed can be achieved by employing the TD. The definition of the TD process can be expressed by

$$
\begin{aligned}
v_{1}(k+1) & =v_{1}(k)+h v_{2}(k), \\
v_{2}(k+1) & =v_{2}(k)+h v_{3}(k), \\
v_{3}(k+1) & =v_{3}(k)+h f s t, \\
f s t & =-r\left(r\left(r\left(v_{1}-\theta_{d}\right)+3 v_{2}\right)+3 v_{3}\right),
\end{aligned}
$$

where $\theta_{d}(t)$ denotes the desired trajectory of the control system, $r$ and $h$ determine the speed and the step length, respectively, and $v_{1}(t), v_{2}(t)$, and $v_{3}(t) \operatorname{track} \theta_{d}(t), \dot{\theta}_{d}(t)$, and $\ddot{\theta}_{d}(t)$, respectively.

With the ESO, the change rate and total disturbance of the error can be real-time estimated using ESO from the control variables and the position errors of the input signal. Thereby, dynamic compensation for the disturbances can be conducted properly using estimated total disturbances. By introducing the novel nonlinear function nfal(.), the improved third-order discrete ESO can be configured as follows:

$$
\begin{aligned}
& e(k)=z_{1}(k)-\theta(k) \\
& z_{1}(k+1)=z_{1}(k)+h\left[z_{2}(k)-\beta_{01} e(k)\right] \\
& z_{2}(k+1) \\
& \quad=z_{2}(k)+h\left[z_{3}(k)-\beta_{02} \operatorname{nfal}\left(e(k), c_{1}, b_{1}, \gamma_{1}\right)\right], \\
& \begin{aligned}
& z_{3}(k+1) \\
&= z_{3}(k) \\
& \quad+h\left[z_{4}(k)-\beta_{03} \operatorname{nfal}\left(e(k), c_{1}, b_{2}, \gamma_{1}\right)+b_{0} u(k)\right], \\
& z_{4}(k+1)=z_{4}(k)-h \beta_{04} \operatorname{nfal}\left(e(k), c_{1}, b_{3}, \gamma_{1}\right),
\end{aligned}
\end{aligned}
$$

where $\beta_{01}, \beta_{02}, \beta_{03}$, and $\beta_{04}$ are adjustable compensation gains, and $a_{1}, a_{2}, a_{3}, \delta_{1}$, and $b_{0}$ are design parameters. $z_{1}(t)$, $z_{2}(t), z_{3}(t)$, and $z_{4}(t)$ are the estimate outputs of the ESO process, respectively, tacking $\theta(t), \dot{\theta}(t), \ddot{\theta}(t)$, and the estimated total disturbance.

The nonlinear function $\mathrm{nfal}(\cdot)$ can be expressed by

$$
\begin{aligned}
\operatorname{nfal}(e(k), c, b, \gamma) & =b \arctan \frac{c(e(k)-\gamma)-\mu_{0}}{\pi / 2-\mu_{0}}, \\
\mu_{0} & =\arctan (c(0-\gamma))
\end{aligned}
$$

where $v$ determines the shape of the operator, $b$ determines the range of the operator, and $\gamma$ determines the center of the operator.

Essentially, the NLSEF controller is a nonlinear PD controller; nonlinear combination of the error components deriving from the outputs of the TD and the state estimation 


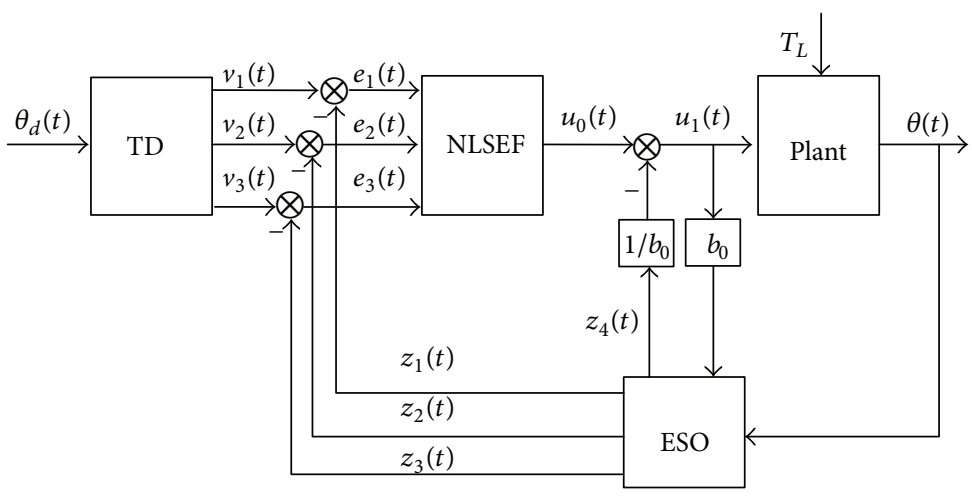

FIGURE 2: Schematics of ADRC.

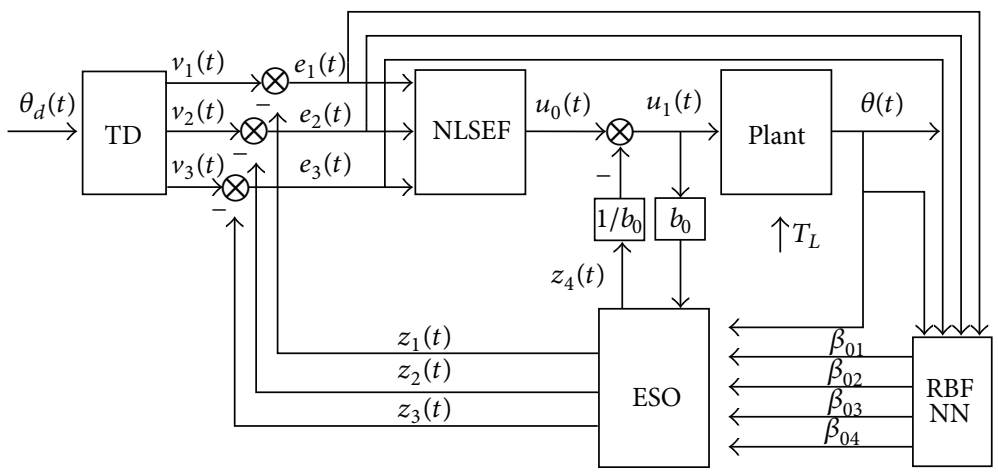

FIgURE 3: The diagram of ADRC based on RBF neural network.

from the ESO is properly designed to construct the command signal $u_{o}(t)$. The third-order discrete governing law of the NLSEF can be determined by

$$
\begin{aligned}
e_{1}(k+1)= & v_{1}(k+1)-z_{1}(k+1), \\
e_{2}(k+1)= & v_{2}(k+1)-z_{2}(k+1), \\
e_{3}(k+1)= & v_{3}(k+1)-z_{3}(k+1), \\
u_{0}(k+1)= & \beta_{1} \operatorname{nfal}\left(e_{1}(k+1), c_{0}, b_{4}, \gamma_{0}\right) \\
& +\beta_{2} \operatorname{nfal}\left(e_{2}(k+1), c_{0}, b_{5}, \gamma_{0}\right) \\
& +\beta_{3} \operatorname{nfal}\left(e_{3}(k+1), c_{0}, b_{6}, \gamma_{0}\right),
\end{aligned}
$$

where $\beta_{1}, \beta_{2}$, and $\beta_{3}$ represent the control gains, respectively, and $a_{4}, a_{5}, a_{6}$, and $\delta_{0}$ are the parameters for the design process.

By combining the estimated disturbance through the ESO, the actual control variables applied to the actuator of the control system can be obtained as follows:

$$
u_{1}(k+1)=\frac{\left[u_{0}(k+1)-z_{4}(k+1)\right]}{b_{0}},
$$

where $b_{0}$ is the compensation factor.

3.2.2. Adaptive Updating of the ESO. Overall, there are four important parameters in the ESO that govern the control accuracy and system robustness of the whole control system, namely, $\beta_{01}, \beta_{02}, \beta_{03}$, and $\beta_{04}$. To achieve optimal control during the whole working process, an online updating of the four parameters based on RBFNN is developed in the present study. The configuration of the adaptive control system is illustrated in Figure 3. As shown in Figure 3, a three-layer RBFNN is adopted where $e_{1}(t), e_{2}(t), e_{3}(t)$, and $\theta(t)$ are serving as the input nodes, while $\beta_{01}, \beta_{02}, \beta_{03}$, and $\beta_{04}$ are serving as the output nodes. The number of node in hidden layer is chosen as 6 .

The goal of adaptive adjustment of the control parameters is to seek for a control signal that can minimize the difference between the process output and the desired output. The performance criterion employed in this paper for the parameter updating is defined by

$$
E(k)=\frac{1}{2}\left[\theta_{d}(k)-\theta(k)\right]^{2}=\frac{1}{2} e^{2}(k) .
$$

The output of each hidden node in the RBFNN can be obtained as follows:

$$
\varphi_{i}(k)=\exp \left(-\frac{\left\|X-C_{i}\right\|^{2}}{2 b_{i}^{2}}\right),
$$

where $X=\left[e_{1}, e_{2}, e_{3}, \theta\right]^{T}$ denotes the input vector of the $\mathrm{NN}, C_{i}=\left[c_{i 1}, c_{i 2}, c_{i 3}, c_{i 4}\right]^{T}$ denotes the center vector of the $i$ th hidden node, and $b_{i}$ is the width of the radial basis function of this node. 


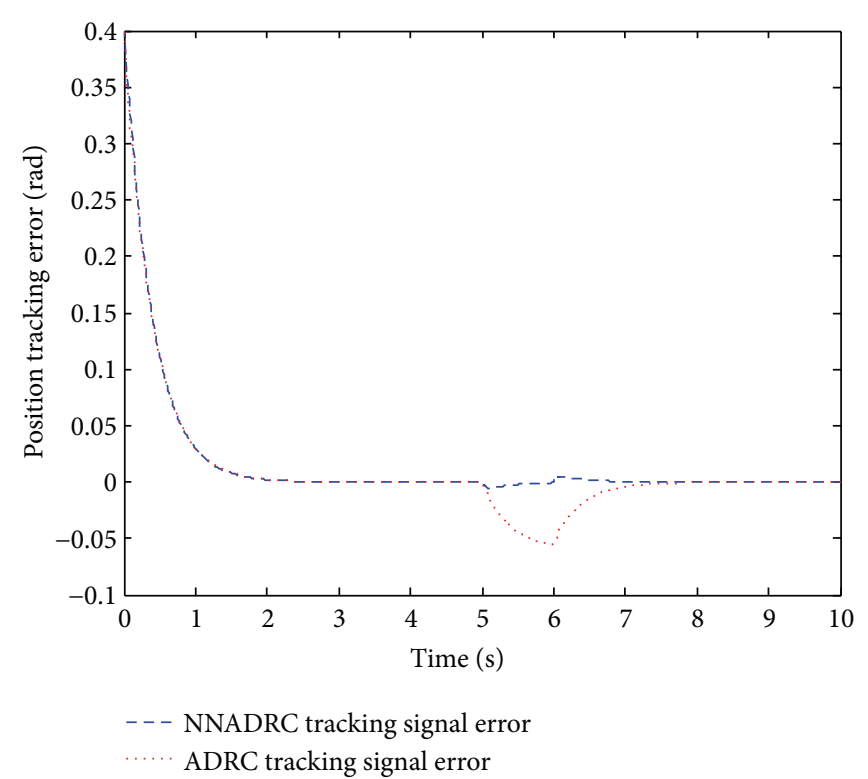

(a)

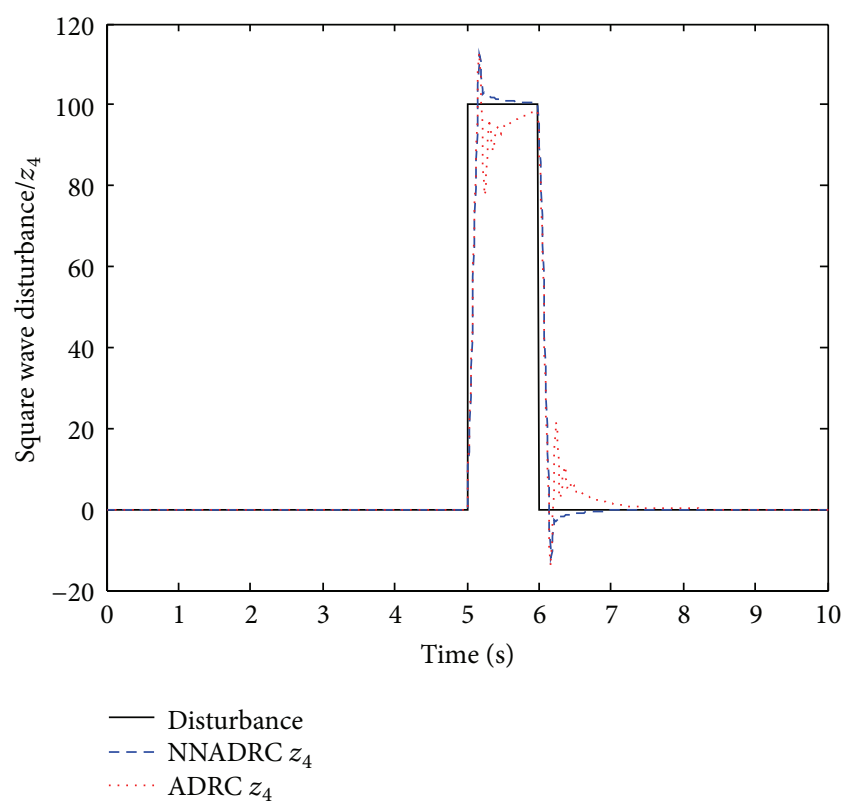

(c)

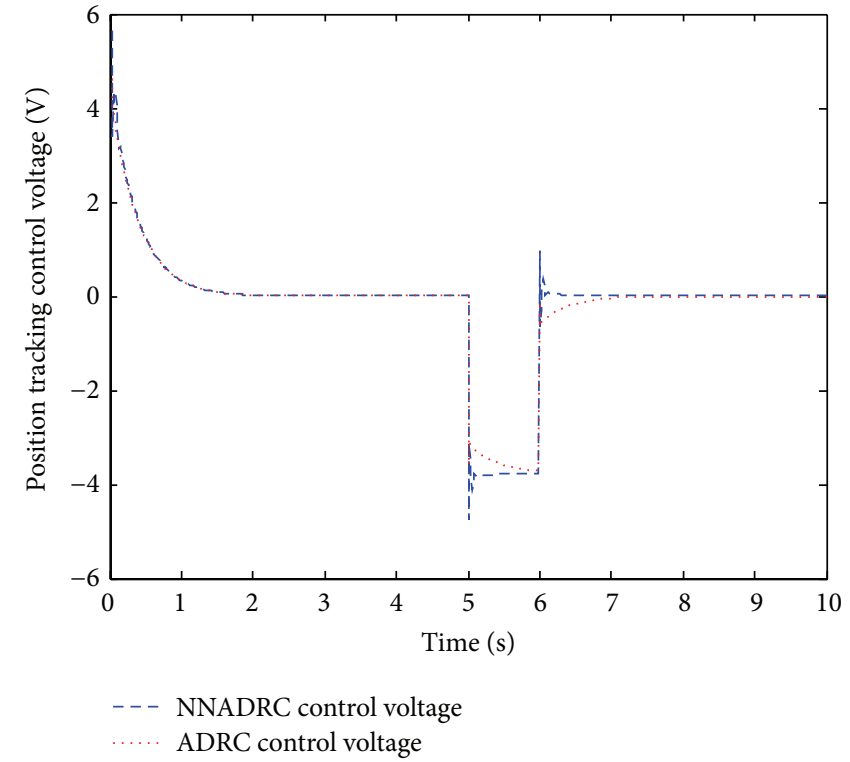

(b)

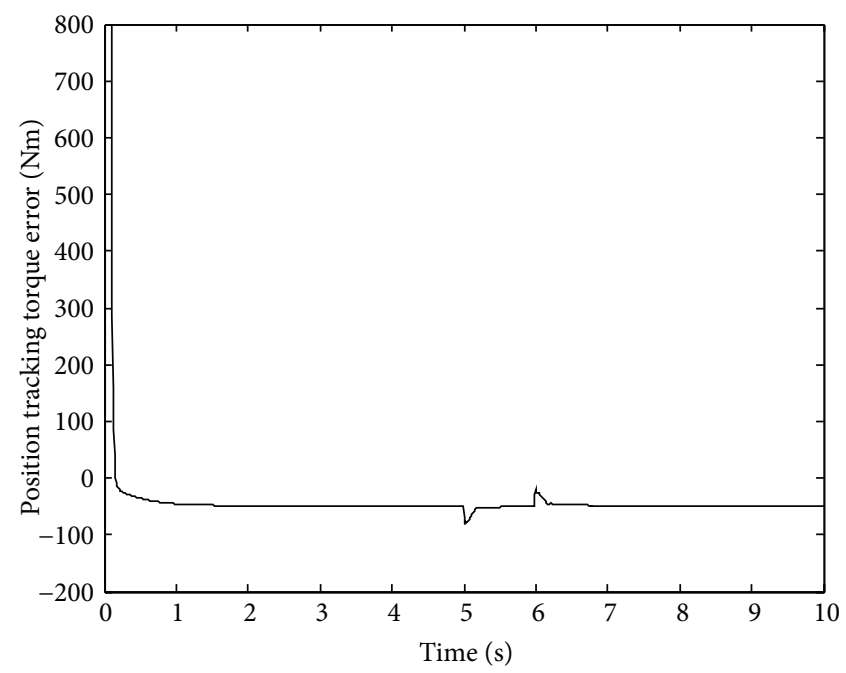

FIGURE 4: Step response of the control system: (a) errors of position signal with square wave disturbance, (b) position tracking control voltage, (c) square wave disturbance and the estimated $z_{4}$, and (d) torque errors of position tracking.

Thus, output of the RBFNN can be determined by

$$
\beta_{0 l}(k)=\sum_{i=1}^{6} \omega_{l i} \varphi_{i}(k)
$$

where $\omega_{l i}$ represents the connection weight between the $i$ th $(i=1,2,3,4)$ hidden node and the $l$ th output node.
The update law for the weights of the RBFNN yields the following:

$$
\begin{aligned}
\omega_{l i}(k) & =\omega_{l i}(k-1)+\Delta \omega_{l i}(k)+\rho \Delta \omega_{l i}(k-1), \\
\Delta \omega_{l i}(k) & =-\eta \frac{\partial E(k)}{\partial \omega_{l i}(k)}=-\eta \frac{\partial E(k)}{\partial \theta(k)} \frac{\partial \theta(k)}{\partial \beta_{0 l}(k)} \frac{\partial \beta_{0 l}(k)}{\partial \omega_{l i}(k)} \\
& =\eta e(k) \frac{\partial \theta(k)}{\partial \beta_{0 l}(k)} \varphi_{i}(k),
\end{aligned}
$$


where $\rho$ and $\eta$ are the momentum and learning factors of this RBFNN, respectively. It is to be noticed that the term $\partial \theta(k) / \partial \beta_{0 l}(k)$ is unknown. Thereby, it will be replaced by $\operatorname{sgn}_{l}(k)$ in practice.

\section{Simulation Results and Discussion}

To demonstrate the effectiveness and superiority of the improved ADRC controller for the EHS system with isoactuation configuration, numerical simulation on step responses and constant velocity tracking of the system was conducted.

In the simulation, parameters for the electrohydraulic servo system were $J=1.55 \times 10^{5} \mathrm{Kg} \cdot \mathrm{m}^{2}, C_{t}=1.5 \times 10^{-13}\left(\mathrm{~m}^{3}\right.$. $\left.\mathrm{s}^{-1}\right) / \mathrm{Pa}, B_{m}=1.35 \times 10^{5} \mathrm{~N} \cdot \mathrm{m} /\left(\mathrm{rad} \cdot \mathrm{s}^{-1}\right), V_{0}=0.011781 \mathrm{~m}^{3}$, $\beta_{e}=700 \mathrm{MPa}$, and $T_{Z}=1.28 \times 10^{5} \mathrm{Nm}$. With the balancing controller, three coefficients for the PID controller were set as $k_{p}=4 \times 10^{-5}, k_{i}=2.3 \times 10^{-6}$, and $k_{d}=3.12 \times 10^{-7}$. Parameters for the TD were $h=0.01$ and $r=0.12$, and those for the NLSEF are set as $\beta_{1}=0.95, \beta_{2}=10.6, \beta_{3}=17.3, c_{0}=0.25$, $b_{4}=2.5, b_{5}=2.0, b_{6}=1.5$, and $\gamma_{0}=0.01$. As for the ESO, the design parameters were set as $c_{1}=0.5, b_{1}=2.5, b_{2}=2.0$, $b_{3}=1.5$, and $\gamma_{1}=0.01$, while the adjustable compensation gains were online updated, and the initial values were set as $\beta_{01}=150, \beta_{02}=40, \beta_{03}=40$, and $\beta_{04}=500$.

4.1. Step Response. Positioning errors of the EHS system in step response obtained by both ADRC and the improved NNADRC controllers are illustrated in Figure 4(a). No overshoots are observed for the two control systems, and the response time for the two control systems at which the positioning errors are within $\pm 0.0005236 \mathrm{rad}(\approx \pm 0.5 \mathrm{mil})$ is about $2.545 \mathrm{~s}$. The steady state error is about $2.7 \times 10^{-4} \mathrm{rad}$. To further evaluate system robustness, external disturbances featuring square wave with an amplitude of $100 \mathrm{KN} \cdot \mathrm{m}$ were added in the control system from $t=5 \mathrm{~s}$ to $t=6 \mathrm{~s}$. From the positioning errors in Figure 4(a), the maximum deviations for ADRC and NNADRC induced by the disturbances are $0.055 \mathrm{rad}$ and $0.005 \mathrm{rad}$, respectively. This indicates that the NNADRC possesses much higher robustness than the ADRC.

The command signal and the estimated total disturbance $\left(z_{4}\right)$ during the step responses are further illustrated in Figures 4(b) and 4(c). As shown in Figure 4(b), much smooth estimation is obtained by the NNADRC controller, showing strong suspension capacity of the chatter phenomenon by adopting the improved nonlinear function $\mathrm{nfal}(\cdot)$. In addition, much more accurate estimation of the total disturbances is achieved in the NNADRC system which may attribute to the real-time updating capacity of the RBFNN. The weighting and balancing torques in the NNADRC system are illustrated in Figure 4(d); a deviation of the torque is about $50 \mathrm{Nm}$. It suggests that the output torque of the balancing cavity finely agrees with the weighting torque by adopting the balancing controller, being capable for active balance of the system weighting torque.

To further investigate system nonlinearity induced performance variation of the control system, parameter perturbation was adopted to mimic the system state deviations caused by the nonlinear effects. Step responses of the ADRC

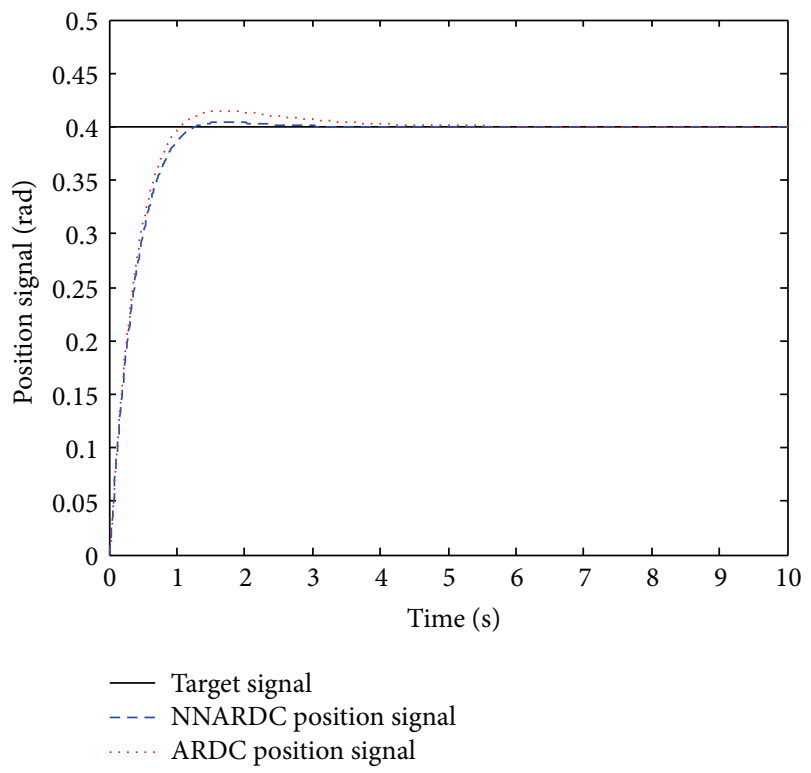

FIGURE 5: ARDC and NNARDC positioning results with $30 \%$ change of $J$.

and NNADRC systems are illustrated in Figure 5 with respect to a variation of the rotational inertia $J$ up to $30 \%$. As shown in Figure 5, the overshoot of the ADRC system is about $0.4147 \mathrm{rad}(3.67 \%)$, and the time for entering the positioning error tolerance is about $5.65 \mathrm{~s}$. In contrast, the overshoot of the NNADRC system is only about $0.1152 \mathrm{rad}(1.06 \%)$ which is only about $28.8 \%$ of that of the ADRC system, and the entering time is about $3.11 \mathrm{~s}$. The parameter perturbation simulation indicates that the NNADRC system has high robustness against parameter perturbation of the control system.

4.2. Constant Velocity Tracking. Constant velocity tracking with $v=0.8727 \mathrm{rad} \cdot \mathrm{s}^{-1}$ was simulated to further investigate performances of the control system. A harmonic disturbance with amplitude and frequency of $A=20 \mathrm{KN} \cdot \mathrm{m}$ and $f=0.5 \mathrm{~Hz}$ is added to simulate external disturbance. The resulting tracking errors obtained by both ADRC and the NNADRC are illustrated in Figure 6(a). As shown in Figure 6(a), the maximum tracking error in the steady state for the NNADRC is about $0.0011 \mathrm{rad}$ which is only about $1 / 9$ that obtained by ADRC. Similarly, the command voltages and estimated total disturbances for the two control systems are, respectively, illustrated in Figures 6(b) and 6(c). Strong fluctuations of the command and estimated signals especially at the initial stage are observed in the ADRC control system, while those of the NNADRC are much smoother. This indicates that the NNADRC well outperform the ADRC control strategy. The weighting and balancing torques in the NNADRC system are illustrated in Figure 6(d). Slight harmonic fluctuation of the torques is observed, and the maximum unbalanced torque is only about $90 \mathrm{Nm}$. The result suggests that the developed EHS system is effective and very 

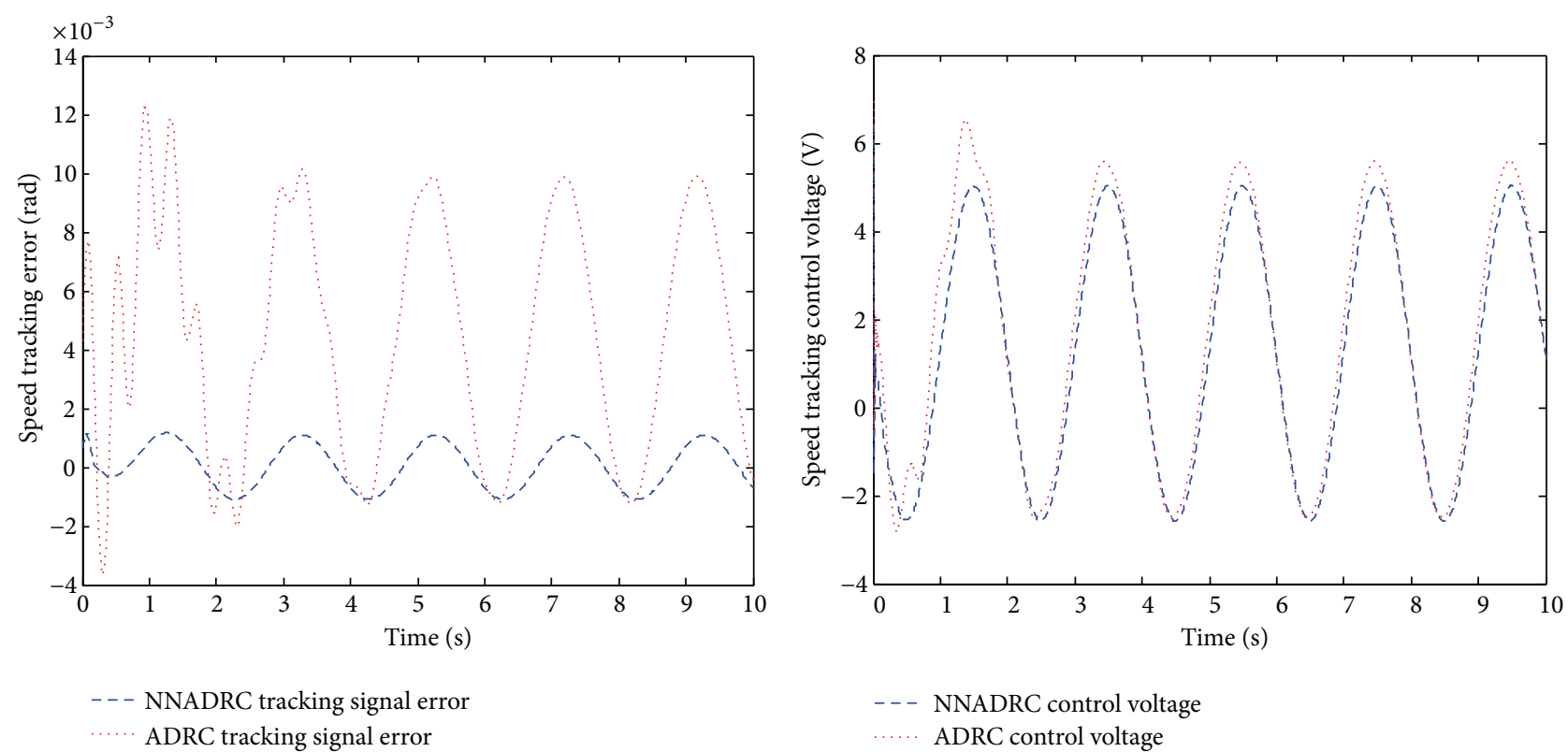

(a)

(b)
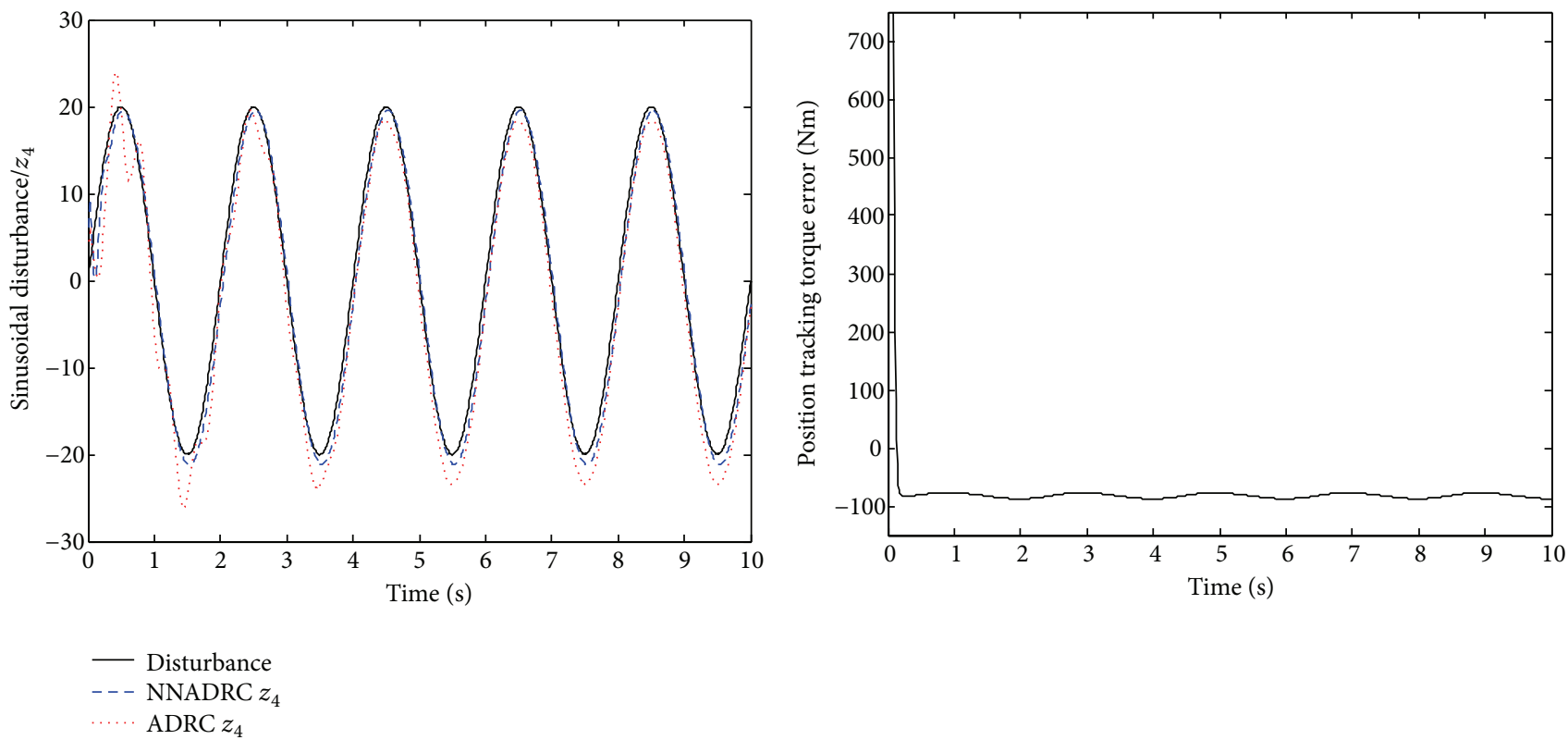

(c)

(d)

FIGURE 6: Response of the control system with constant speed tracking. (a) Errors of speed signal with sinusoidal disturbance, (b) speed tracking control voltage, (c) sinusoidal disturbance and the estimated $z_{4}$, and (d) torque errors of speed tracking.

promising for simultaneous balancing and positioning of large pipes.

\section{Conclusions}

To satisfy the lightweight requirements of large pipe weapons, a novel electrohydraulic servo (EHS) system where the hydraulic cylinder possesses three cavities is developed and investigated in the present study. In the EHS system, the balancing cavity of the EHS is especially designed for active compensation for the unbalancing force of the system, whereas the driving cavity is employed for the positioning and disturbance rejection of the large pipe. By adopting the isoactuation configuration, a more compact and lightweight gun control system can be achieved.

Aiming at simultaneous balancing and positioning of the EHS system, a novel neural network based active disturbance rejection control (NNADRC) strategy is developed. In the NNADRC, the radial basis function (RBF) neural network is employed for online updating parameters of the extended state observer (ESO). By online updating of the estimator 
gains of the ESO, the nonlinear behavior and external disturbance of the system can be accurately estimated and compensated in real time.

The efficiency and superiority of the control system are critically investigated by conducting numerical simulations. When suffering square disturbance in step response, the positioning error obtained by NNADRC is only $10 \%$ of that obtained by ADRC, and the overshoot and entering time of the NNADRC are about $28.8 \%$ and $55 \%$ of them in ADRC system when encountering parameter perturbation, respectively. Meanwhile, when suffering harmonic disturbance in constant speed tracking, the steady accuracy is enhanced by 9 times. In addition, the inherent chatter phenomenon in ADRC can also be well suspended by adopting the improved nonlinear function scheme in the NNADRC.

\section{Conflict of Interests}

The authors declare that there is no conflict of interests regarding the publication of this paper.

\section{Acknowledgment}

The work described in this paper was supported by the National Natural Science Foundation of China (51305205).

\section{References}

[1] D. J. Purdy, "Comparison of balance and out of balance main battle tank armaments," Shock and Vibration, vol. 8, no. 3-4, pp. 167-174, 2001.

[2] V. Marcopeli, M. Ng, and C. Wells, "Robust control design for the elevation axis stabilization of the M256E1 long gun," Report of Defense Technical Information Center of USA, Defense Technical Information Center, Fort Belvoir, Va, USA, 2001.

[3] Q. Gao, Z. Sun, G. L. Yang, R. Hou, L. Wang, and Y. Hou, "A novel active disturbance rejection-based control strategy for a gun control system," Journal of Mechanical Science and Technology, vol. 26, no. 12, pp. 4141-4148, 2012.

[4] C. Ma and X. Zhang, "Simulation of contamination prevention for optical window in laser ignition systems of large-caliber guns," Journal of Applied Mechanics, vol. 78, no. 5, Article ID 051014, 7 pages, 2011.

[5] Ö. Gümüşay, Intelligent Stabilization Control of Turret Subsystems under Disturbances form Unstructured Terrain, Middle East Technical University, 2006.

[6] T. Karayumak, Modeling and stabilization control of a main battle tank [Ph.D. thesis], Middle East Technical University, Ankara, Turkey, 2011.

[7] C. W. Han, The Robust Control and Application of Artillery Position Servo System, Xi'an Jiaotong University, 2002.

[8] J. Q. Han, "Auto disturbances rejection controller and it's applications," Control and Decision, vol. 13, no. 1, pp. 19-23, 1998.

[9] J. Q. Han, "From PID technique to active disturbances rejection control technique," Control and Decision, vol. 9, no. 3, pp. 13-18, 2002.

[10] J. Q. Han, "From PID to active disturbance rejection control," IEEE Transactions on Industrial Electronics, vol. 56, no. 3, pp. 900-906, 2009.
[11] W. Y. Chen, F. L. Chu, and S. Z. Yan, "Stepwise optimal design of active disturbances rejection vibration controller for intelligent truss structure based on adaptive genetic algorithm," Journal of Mechanical Engineering, vol. 46, no. 7, pp. 74-81, 2010.

[12] H.-P. Ren and F. Zhu, "Optimal design of speed adaptive disturbance rejection controller for brushless DC motor based on immune clonal selection algorithms," Electric Machines and Control, vol. 14, no. 9, pp. 69-74, 2010.

[13] X.-Q. Liu, L. Tang, and L.-T. Zhu, “Three-motor synchronous control system based on fuzzy active disturbances rejection control," Electric Machines and Control, vol. 17, no. 4, pp. 104109,2013

[14] G.-L. Qiao, C.-N. Tong, and Y.-K. Sun, "Study on mould level and casting speed coordination control based on ADRC with DRNN optimization," Acta Automatica Sinica, vol. 33, no. 6, pp. 641-648, 2007.

[15] X.-H. Qi, J. Li, and S.-T. Han, "Adaptive active disturbance rejection control and its simulation based on BP neural network," Acta Armamentarii, vol. 34, no. 6, pp. 776-782, 2013.

[16] K. C. Li, "Adaptive auto disturbance rejection control for servo systems based on the RBF neural network," Electrical Automation, vol. 32, no. 2, pp. 23-25, 2010.

[17] Z. Y. Liu, W. Liu, M. Y. Fu et al., "Trace-keeping of an air cushion vehicle based on an auto disturbance rejection controller with a recurrent networks model," Journal of Harbin Engineering University, vol. 33, no. 3, pp. 283-288, 2012.

[18] X. T. Li, B. Zhang, J. H. Sun, D. Mao, X. Bai, and H. Shen, "ADRC based on disturbance frequency adaptive of aerial photoelectrical stabilized platform," Infrared and Laser Engineering, vol. 43, no. 5, pp. 1574-1581, 2014.

[19] Y.-Y. Ma, S.-J. Tang, J. Guo, and J. Shi, "High angle of attack control system design based on ADRC and fuzzy logic," Systems Engineering and Electronics, vol. 35, no. 8, pp. 1711-1716, 2013.

[20] Y. A. Feng, X. P. Zhu, and Z. Zhou, "Adaptation cascade active disturbance rejection controller for flexible flying wing UAV attitude control," Infrared and Laser Engineering, vol. 43, no. 5, pp. 1594-1599, 2014. 


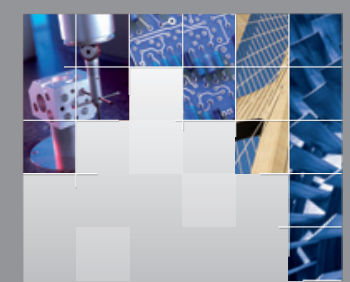

\section{Enfincering}
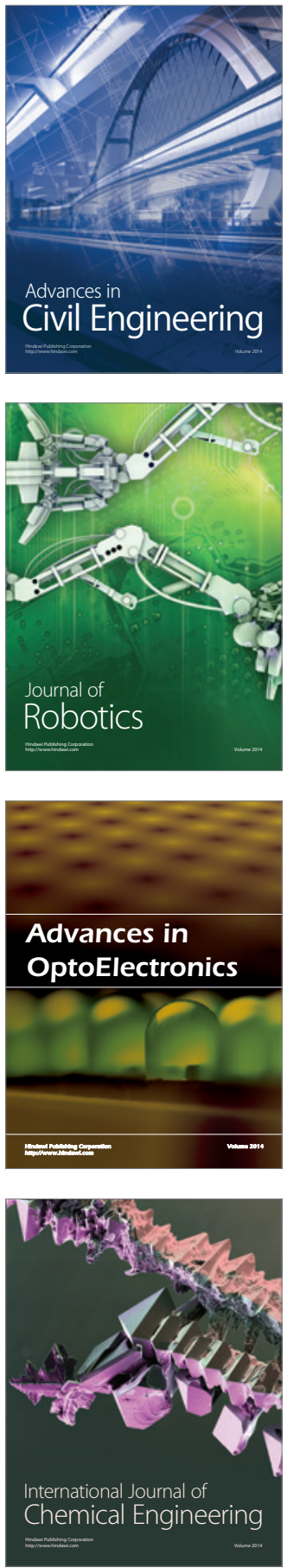

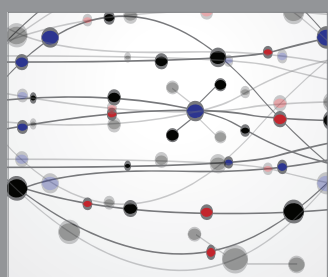

The Scientific World Journal

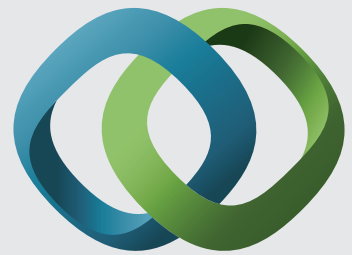

\section{Hindawi}

Submit your manuscripts at

http://www.hindawi.com
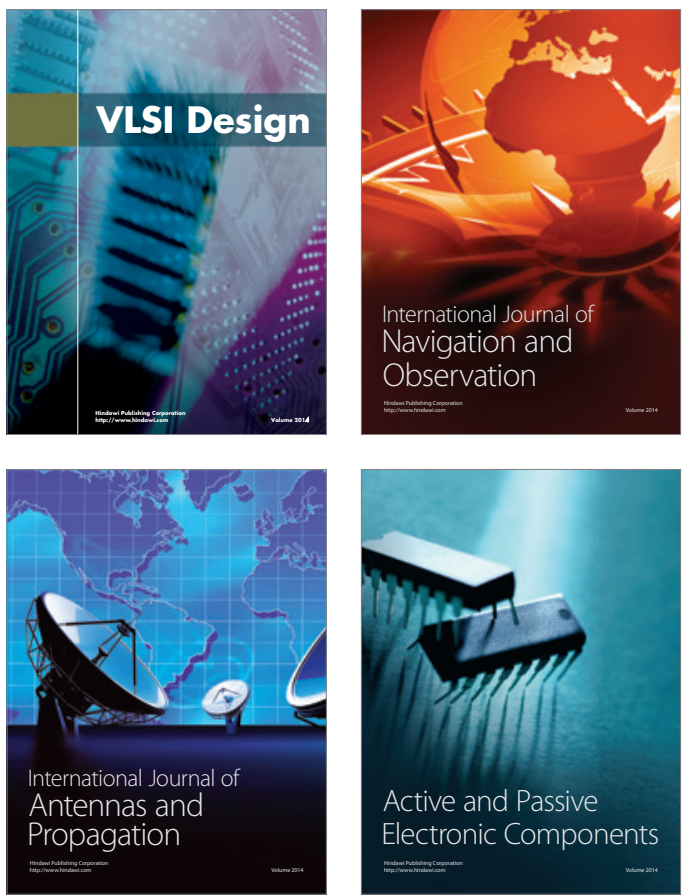
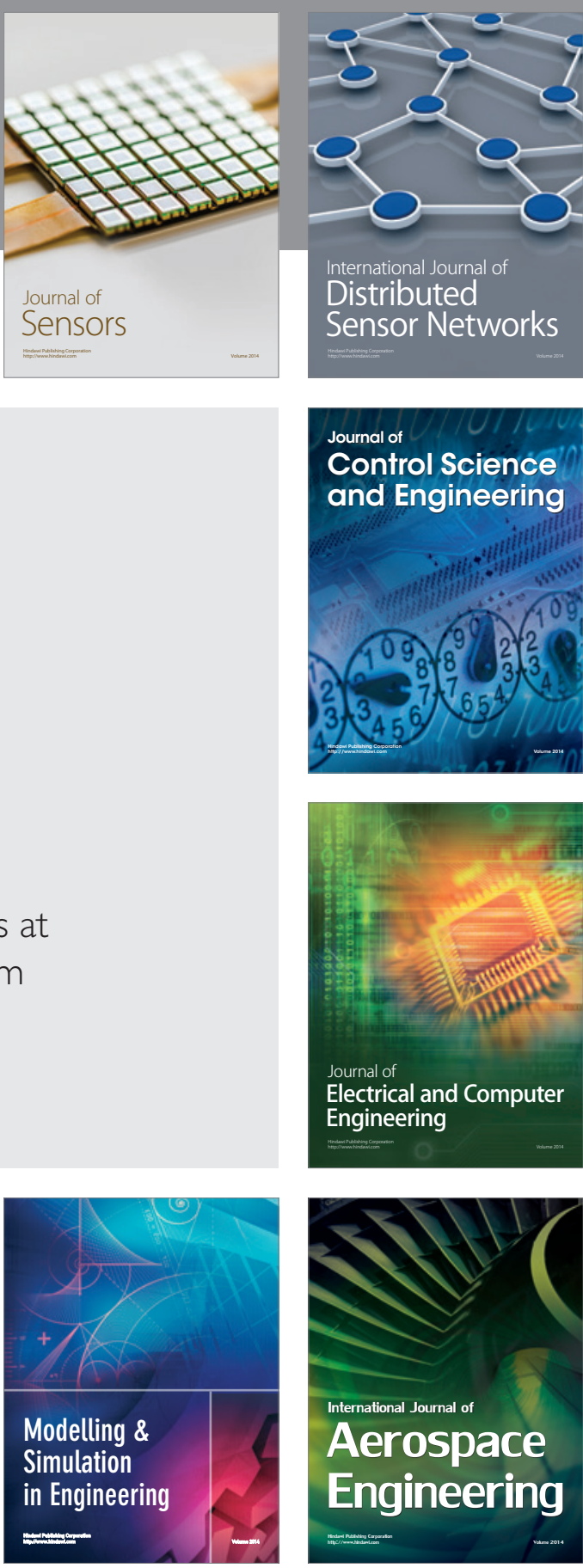

International Journal of

Distributed

Sensor Networks

Journal of

Control Science

and Engineering
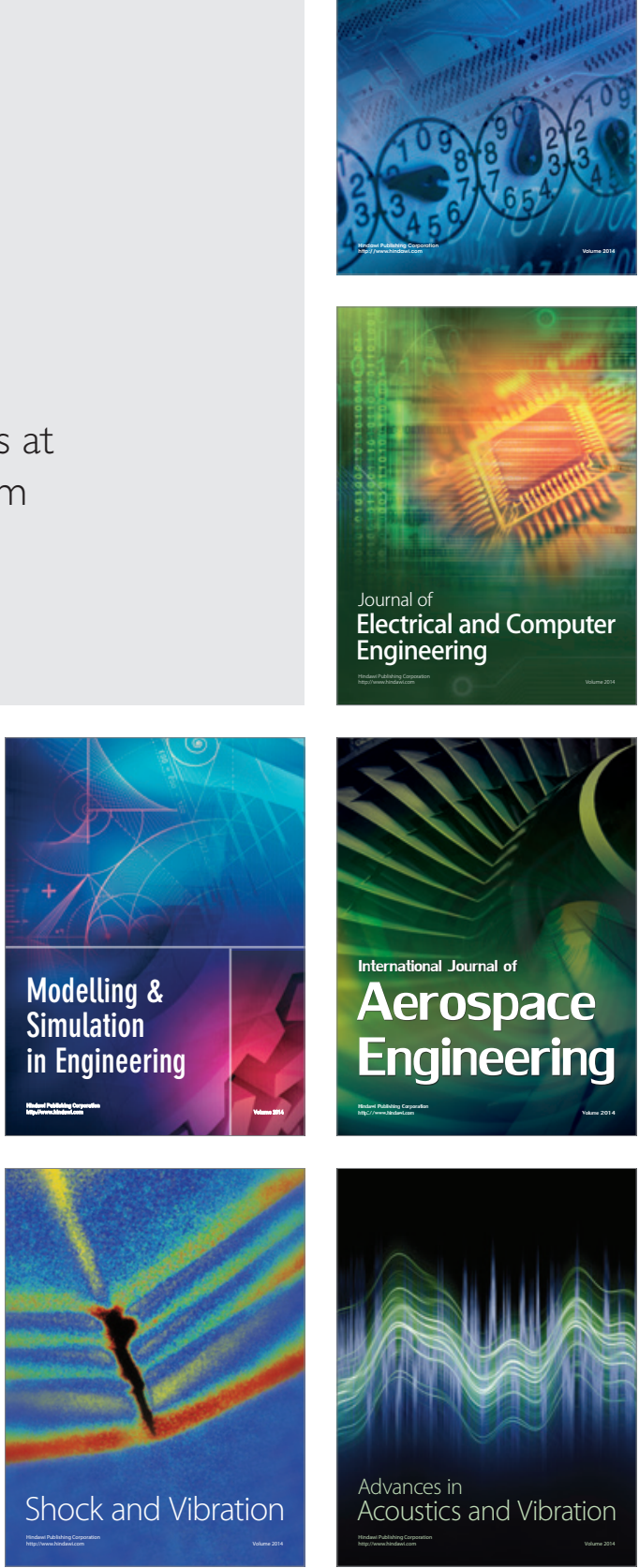